Hanna Dmeńska', Małgorzata Pac ${ }^{2}$, Małgorzata Skomska-Pawliszak², Barbara Pietrucha², Beata Wolska-Kuśnierz'르, Barbara Piątosa ${ }^{3}$, Justyna Komarnicka ${ }^{4}$, Edyta Heropolitańska-Pliszka ${ }^{2}$

'The Pulmonology Outpatients' Clinic, The Children's Memorial Health Institute, Warsaw, Poland

${ }^{2}$ Department of Immunology, The Children's Memorial Health Institute, Warsaw, Poland

${ }^{3}$ Histocompatibility Laboratory, The Children's Memorial Health Institute, Warsaw, Poland

${ }^{4}$ Department of Radiology and Diagnostic Imaging, The Children's Memorial Health Institut, Warsaw, Poland

\title{
Progressive bronchiectasis and CMC in a patient with STAT1 GOF — a rare case of primary immunodeficiency
}

\begin{abstract}
Bronchiestasis is a common complication developing in patients with primary immunodeficiency disorders. AD GOF STAT1 deficiency is characterized by CMC, repeated infections, and autoimmunity. It is the most frequently diagnosed entity in a group of PIDs with CMC. Here, we present the first Polish case of a female patient with early-onset bronchiestasis accompanied by CMC and a severe course of infections who was genetically diagnosed with AD GOF1 STAT1 mutation at the age of 15.
\end{abstract}

Key words: primary immunodeficiency, bronchiectasis, STAT1 GOF, CMC

Adv Respir Med. 2020; 88: 271-277

\section{Introduction}

Bronchiectasis is a chronic respiratory disease characterized by an abnormal permanent dilation of the bronchi which are typically described as cylindrical, varicose, or cystic in appearance. The condition is characterized by a vicious cycle of persistent bacterial infections and excessive neutrophilic inflammation leading to the impairment of airway defence mechanisms. Risk factors for bronchiectasis are listed in Table 1 [1]. Appropriate baseline investigations include a chest radiograph, pulmonary function tests (PFT), and sputum bacteriological cultures. However, the gold standard for confirming the diagnosis is high-resolution computed tomography (HRCT) of the chest. The main aims of management are to reduce symptoms and exacerbation frequency and severity, to preserve lung function, and to improve the patient's health-related quality of life [2].

Bronchiectasis is also a well-recognized complication of primary antibody deficiencies (PAD), which are the most frequently diagnosed primary immunodeficiency disorders (PID). PAD patients share a significant susceptibility to respiratory diseases that represent a relevant cause of morbidity and mortality. Pulmonary complications include acute and chronic infection-related diseases such as pneumonia and bronchiectasis, and immunemediated interstitial lung diseases such as granulomatous-lymphocytic interstitial lung disease (GLILD) and cancer [3]. A recent review of the UK PID Registry data showed that $47 \%$ of a cohort of 801 patients with primary hypogammaglobulinemia had bronchiectasis confirmed by HRCT [4]. Unfortunatelly, lung disease can progress in PID patients despite conventional treatment with immunoglobulin G (IgG) replacement therapy and/or antibiotic prophylaxis [4].

High susceptibility to mucosal and skin candidiasis is a hallmark of several PIDs associated with impaired interleukin-17 (IL-17) $\mathrm{T}$ cell immunity. Isolated chronic mucocutaneous candidiasis (CMC) was first described in the 1960s, but its genetic causes are still being

Address for correspondence: Edyta Heropolitańska-Pliszka, Department of Immunology, The Children's Memorial Health Institute, Warsaw, Poland; e-mail: ehp@poczta.onet.eu DOI: 10.5603/ARM.2020.0112

Received: 24.11.2019

Copyright (C) 2020 PTChP

ISSN 2451-4934 
Table 1. Causes of bronchiectasis with focus on primary immunodeficiencies

\begin{tabular}{lc}
\hline Cause & Mean incidence \\
\hline Primary immunodeficiency disorders & $1.1-16.0 \%$ \\
— common variable immunodeficiency & \\
$\quad$ (CVID) & \\
— agamaglobulinaemia (XLA and AR) & \\
— hyper-IgM syndromes (HIGM) & \\
— autosomal dominant Hyper-IgE Syn- & \\
$\quad$ drome (AD HIES) & \\
Cystic fibrosis & $0.6-2.7 \%$ \\
Alpha-1 antitrypsin deficiency & $0.6-11.3 \%$ \\
Primary ciliary dyskinesia & $2.0-10.3 \%$ \\
Allergic bronchopulmonary aspergillosis & $0.9-7.8 \%$ \\
Autoimmune/connective tissue diseases & $1.8-31.1 \%$ \\
(typically rheumatoid arthritis, SLE) & \\
Inflammatory bowel diseases & $1.0-3.0 \%$ \\
Congenital malformations & $0.2-0.6 \%$ \\
Aspiration & $0.2-11.3 \%$ \\
Postinfectious & $29.0-42.0 \%$ \\
Idiopathic* & $26.0-53.0 \%$ \\
\hline
\end{tabular}

*0ther causes excluded

investigated. In 2011, a group of scientists from the Laboratory of Human Genetics of Infectious Diseases (Necker Hospital, Paris) reported 47 patients with autosomal dominant (AD) CMC from 20 kindreds with 12 gain-of-function (GOF) mutations in the signal transducer and activator of transcription 1 (STAT1) gene [5].

\section{Case report}

Here, we report the first Polish case of a 19-year-old female with a STAT1 GOF pathogenic variant who presented with recurrent respiratory bacterial infections complicated by CMC and severe bronchiectasis. She was born as a second child to young, healthy and unrelated parents after an uneventful pregnancy and delivery, and without a family history of recurrent infections. She received the BCG vaccine during the first days of life. At the 2nd week of age, she was treated with intravenous antibiotics due to multiple abscesses of the scalp. During her first year of life, she was hospitalized for pneumonia twice with accompanied persistent oral thrush (Candida glabrata) and severe malnutrition. Infection with typical and atypical bacteria, viruses (CMV), alpha-1 antitrypsin deficiency, cystic fibrosis, and congenital malformations of the respiratory system were excluded. Performed chest computed tomography (CT) revealed inflammatory changes with atelectasis in segment (S) 2 and in the lower lobe of the right lung. Despite the fact that the presence of acid-fast bacilli (AFB) in bronchial lavage was not confirmed, clinical course indicated tuberculosis. During anti-mycobacterial therapy (rifampicin - RMP, isoniazid - INH, streptomycin - SM, pyrazinamide - PZA) lasting for 6 months, systematic clinical improvement was observed with resolution of inflammatory lesions in a second chest CT. At the age of 4 , another chest CT was performed due to a chronic cough and periodic bronchopulmonary exacerbations. The results of this CT showed a progression of changes including linear densities in S1 and S2 of the right lung, bilateral striatal-atelectical lesions in S4 and S5, thickening of bronchial walls, and bronchiectasis in S8, S9, and S10 of the left lung. Colonization of airways with Pseudomonas aeruginosa and methicillin-resistant Staphylococcus aureus (MSSA) has been observed since then. Since the age of 7 , the patient has been suspected of having PID with CMC. Physical examination revealed severe body mass and growth delay (BMI $13 \mathrm{~kg} / \mathrm{m}^{2}$ ), clubbing, oral thrush, dental caries, splenomegaly, as well as crackling and rhonchi over both lungs. Immunological tests showed $\mathrm{IgA}, \mathrm{IgG}_{2}$, and $\mathrm{IgG}_{4}$ deficiency, mild CD4+ lymphopenia, reversed CD4/CD8 ratio, and a very low percentage and count of CD19+ cells (Table 2). Initially, common variable immunodeficiency (CVID) was suspected. This was then changed to combined immunodeficiency (CID) and intravenous immunoglobulin supplementation (IVIG), antibiotic and antifungal prophylaxes were recommended. Despite state-of-the-art treatment, the patient still suffered from severe recurrent bacterial and muco-cutaneous fungal (Candida spp) infections. Destructive changes in the respiratory tract continued. At the age of 10 , she was operated on to treat a severe hiatus hernia. Progressive esophageal obstruction associated with post-inflammatory stenosis of the esophagus (most likely a consequence of chronic candidiasis) required numerous esophageal dilatations. At the age of 13, molecular tests were performed in the Necker Hospital in Paris. Two years later, we were informed about heterozygous missense mutations in STAT1 leading to deleterious amino acid substitutions in the DNA binding domain (P1: c.1154C > T; p.T385M) which provided us with a definitive diagnosis for the patient. The parents of the patient were not genetically evaluated. At the age of 15 , she 


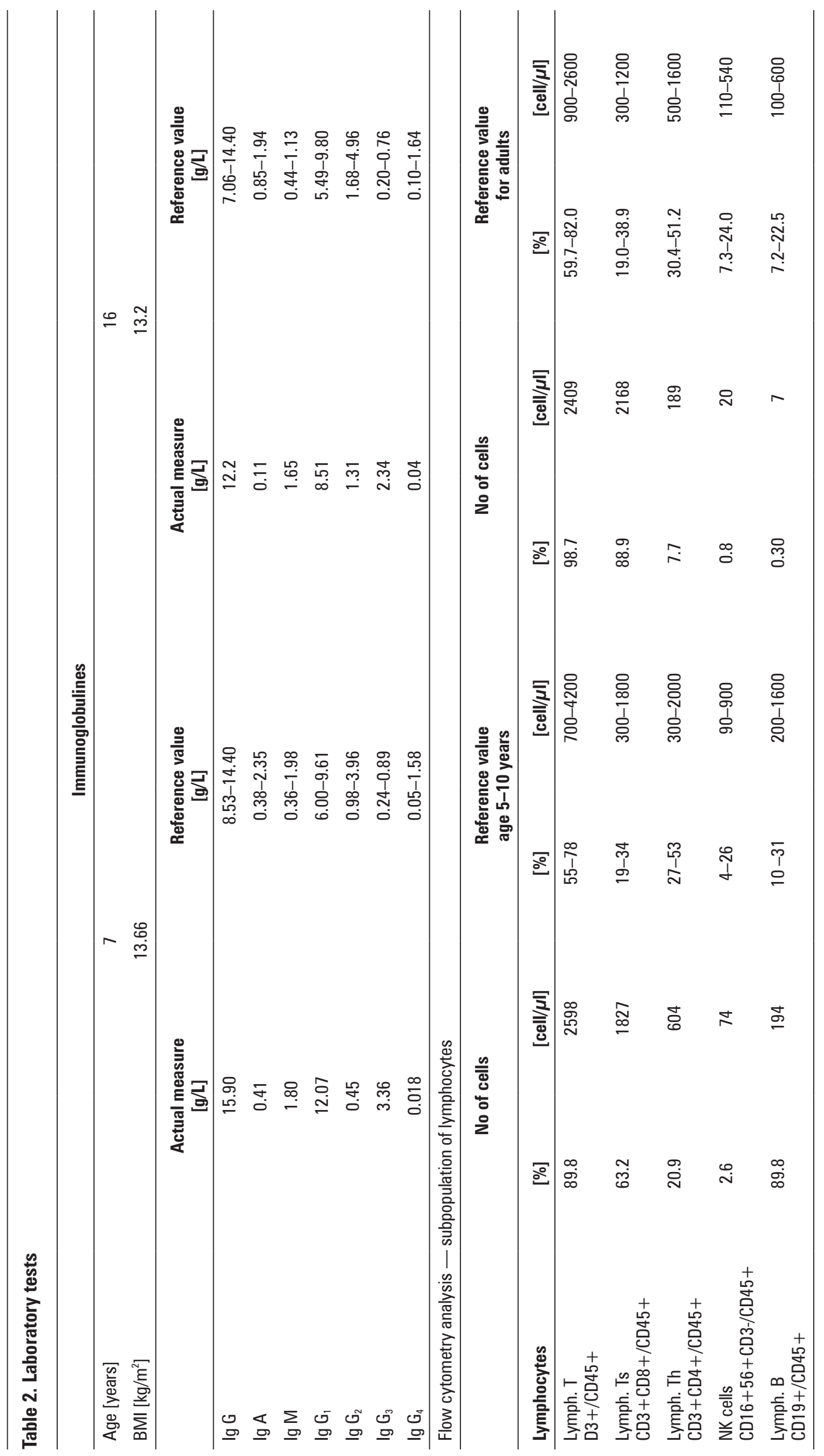


developed a severe invasive skin and pulmonary bacterio-fungal infection which was resistant to fluconazole. Chronic treatment with voriconazole has been implemented since then. At this time, CT scans show a significant degree of cylindrical and cistic bronchiectases in both lungs (Figure 1). Due to hypothyroidism, supplementation of thyroid hormones was incorporated. During the 11-year-follow-up, PFTs were repeatedly performed and an obstructive ventilatory pattern was present increasing airway resistance. Air trapping was gradually worsening. However, difficult cooperation of the patient influcenced PFT quality and brought interpreting difficulties.

At the age of 16, hematopoietic stem cell transplantation (HSCT) was considered due to a progressively deteriorating clinical and immunological condition of the patient (Table 2). However, the unwillingness of patient and her parents towards the procedure together with severe lung disease and the poor worldwide success rates of HSCT discouraged us from this decision. Currently, the patient is treated with subcutaneous immunoglobulins (SCIG), an antifungal agent (voriconazole), and antibacterials (Co-trimoxazole, macrolides, inhaled colistine) for prophylaxis.

\section{Discussion}

STAT1 is the target of heritable loss-of-function (LOF) or gain-of-function (GOF) mutations that give rise to distinct clinical phenotypes. STAT1 mediates the actions of many cytokines involved in mounting innate and adaptive immune responses to viruses and intracellular bacteria [5, 6]. Recurrent bacterial infections are common to both types of STAT1 mutations. AR
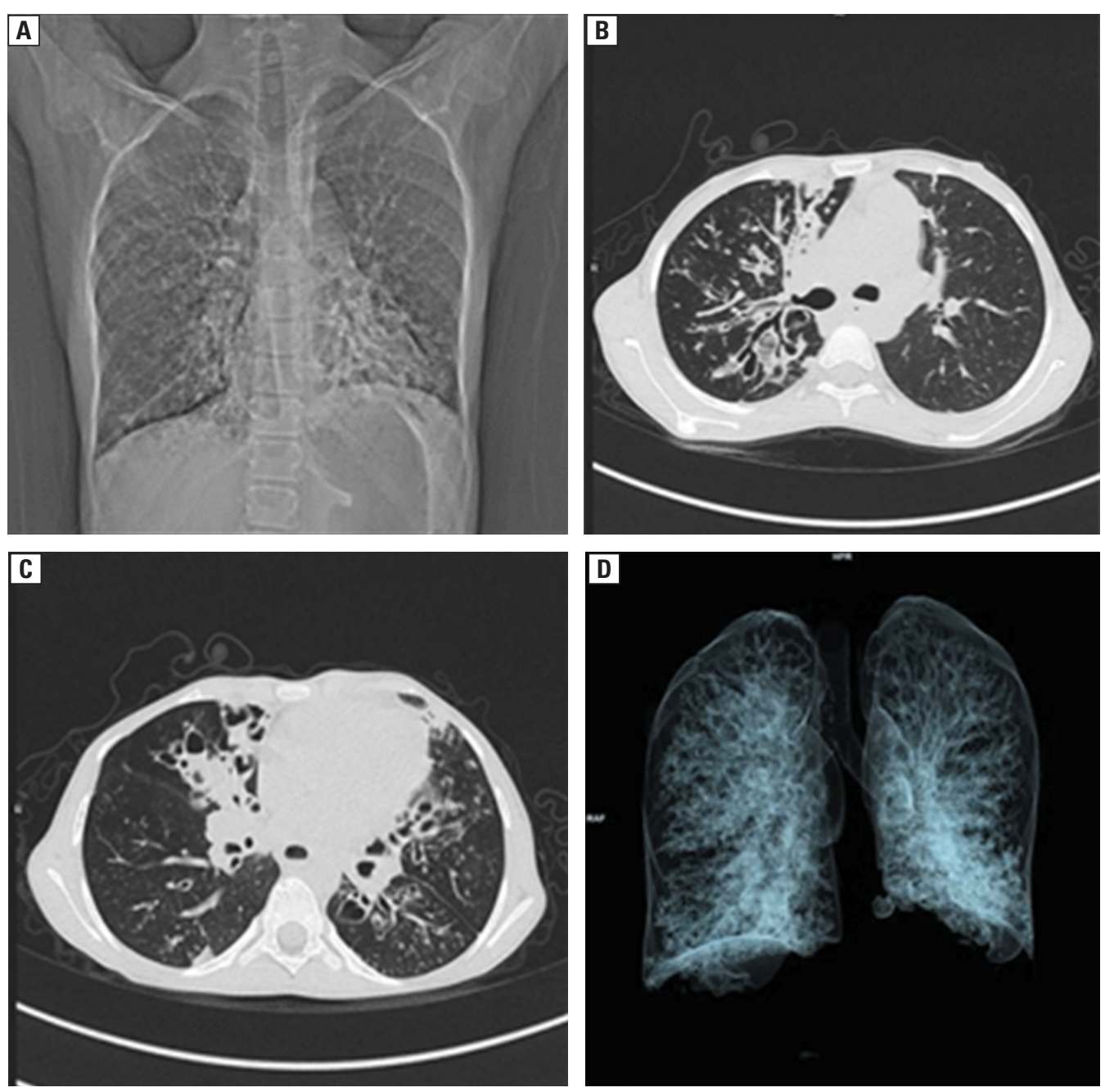

Figure 1. High-resolution computed tomography of the chest. A. Frontal plane; B. Cystic bronchiectasis with thickening of bronchial walls; C. Saccular dilatation of bronchi, partly with mucoid impaction in some places; D. three-dimensional imaging — a significant degree of cylindrical and cystic bronchiectasis in both lungs 
LOF mutations are additionally defined by invasive mycobacteriosis and viral infections, especially Herpesviridae (HSV, CMV, EBV), whereas AD LOF mutations present with susceptibility to mycobacteria but normal immunity against viruses. AD GOF mutations are distinguished by chronic mucocutaneous candidiasis and autoimmunity [5, 7].

GOF mutations in STAT1 have increasingly been identified as a genetic cause of AD CMC which impaires the development of IL-17-producing $\mathrm{T}$ cells $[5,6]$. CMC is a heterogeneous disorder with recurrent chronic Candida spp infections primarily involving the nails, skin, and oropharynx. CMC can be associated with various pathological conditions listed in Table 3. Recent studies have demonstrated that the clinical spectra of STAT1 GOF mutations continue to expand encompassing bronchiectasis, immune dysregulation, and combined immunodeficiency [8,9]. Disease manifestations of GOF STAT1 can be mild or severe and life-threatening $[9,10]$.

Here, we describe a patient with STAT1 GOF mutations who experienced poor weight gain, chronic refractory candidiasis, recurrent pneumonia resulting in bronchiectasis, and severe oral and esophageal candidiases with strictures associated with hypothyroidism. The clinical picture of our patient was consistent with a combined immunodeficiency phenotype resembling a few cases reported in literature $[11,12]$. The authors described two patients presenting in early infancy with candidiasis and chronic lung findings including bronchiectasis. One of them also developed a mycobacterial infection.

STAT1 GOF mutations may present early in life with very complex and variable phenotypes. Patients, in most cases, suffer from persistent and recurrent infections of the skin, nails, and mucosa, mainly caused by C. albicans, variably associated with bacterial (i.e., respiratory tract and skin), viral (i.e., mostly Herpesviridae), and, less frequently, with mycobacterial infections. They also present with susceptibility to autoimmune diseases (i.e., hypothyroidism, type 1 diabetes, blood cytopenia, systemic lupus erythematosus, vitiligo), enteropathy, cardiac and vascular alterations, bronchiectasis, parodontitis, and failure to thrive [5, 6]. Frequent infectious events increase the risk of chronic lung disease with a severe impact on the quality of life of these patients [7]. Chronic-recurrent infections with different pathogens leading to significant morbidity suggest combined immunodeficiency, CMC, or Mendelian susceptibility to mycobacterial diseases.
Table 3. Primary immunodeficiencies connected with CMC

$A D$ gain-of-function mutation in signal transducer and activator of transcription 1 (GOF STAT1)

Severe combined immunodeficiency (SCID)

Combined immunodeficiencies (CID)

Combined immunodeficiency-like dedicator of cytokinesis 8 deficiency (DOCK8)

$A D$ hyper-IgE syndrome (AD-HIES) - signal transducer and activator of transcription 3 (STAT3) deficiency

Autoimmune polyendocrine syndrome type 1 (APS-1)

IL-12R $\beta 1$ and IL-12p40 deficiencies

IL17F, IL17RA, IL17RC

Tyrosine kinase 2 (TYK2) deficiency

Caspase recruitment domain 9 (CARD9)

In 2016, an international multicenter study examined 274 patients with AD STAT1 GOF mutations. 98\% had CMC with a median age at onset of 1 year, $74 \%$ had bacterial infections with recurrent lobar pneumonia, bronchitis or interstitial pneumonia (47\%), mainly caused by Streptococcus pneumoniae, Pseudomonas aeruginosa, Haemophilus influenzae and Staphylococcus aureus, but only 17 patients ( $6 \%$ of the studied patients) had a mycobacterial infection. Bronchiectasis was reported in $21 \%$ of the 274 patients [13]. On one hand, chronic and/or recurrent mucocutaneous fungal infections with predominantly C. albicans are the major infectious complications in patients with STAT1 GOF mutations and generally arise in infancy or childhood. On the other hand, the recurrence of pulmonary exacerbations frequently leads to chronic lung disease with an obstructive component as shown by spirometry and progresses to bronchiectasis in childhood. Additionaly colonization with Pseudomonas aeruginosa is associated with an increased rate of decline in lung function and a poorer health-related quality of life. That is why careful consideration to the possibility of STAT1 GOF mutations should be given at the time of CMC diagnosis since they are reported to be causative in more than half of CMC patients [13]. Still, we should bear in mind that these patients may exhibit quite different phenotypes, new infections, bronchiectasis, autoimmune diseases, malignancies, and aneurysms that may emerge gradually with age.

Most patients (70-80\%) present with normal lymphocyte ratios, immunoglobulin levels, and T-cell function despite the high rate of infections. However, some of the STAT1 GOF mutated patients may develop $\mathrm{T}(\mathrm{CD} 3+)$ and, more frequ- 
ently, B (CD19+) cell lymphopenia and hypogammaglobulinemia [7, 13], which was also observed in a described patient above. In STAT1 GOF patients, B cell deficiency has been variably reported with loss of both switched and unswitched memory B cells with age which, together with a low concentration of immunoglobulins, may suggest CVID. This disease-bound progressive loss of immunologic function despite appropriate treatment (antibacterial, antifungal prophylaxis and immunoglobulins substitution) may be another possible reason for newly acquired infections as well as explain the progression of lung disease, which our patient experienced. CVID was also the first provisional diagnosis which was given to the reported patient after a wide range of immunological tests performed for the first time. Recently, a STAT1 GOF mutation has been detected in a patient with a diagnosis of CVID, CMC, and delayed neurocognitive development in association with Th17 deficiency and low Treg counts [14]. That is why most of patients are initially followed with various immunological diagnoses such as CID, IPEX-like syndrome and even undergo HSCT before identification of the molecular defect.

It was reported that phenotypic manifestations of the various STAT1 mutations may differ despite the similar molecular and cellular/immunological features. However, Uzel et al. [15], Soltesz et al. [16], and Sampaio et al. [17] reported patients with the same c.1154C > T (p.Thr385Met) GOF STAT1 mutation as found in our patient. These patients presented with CMC, recurrent lower respiratory tract infections, bronchiectasis, and autoimmunities similar to the case described here. That being said, more clinical information and phenotype-genotype studies are required to define clinical phenotype caused by AD STAT1 GOF.

Management of patients with STAT1 GOF mutations should target prevention and treatment of infections. Prophylactic antimicrobials and IVIG are routinely used for this purpose. JAK (Janus kinase) inhibitors may potentially be useful in some patients as adjunct therapy pending definitive treatment with HSCT [18]. The fact that CMC is heterogeneous, progressive, and unpredictable in its course should alert physicians to recognize early HSCT as a feasible treatment option to avoid severe morbidity and mortality. However, HSCT is variable in success because despite being curative, it brings with it a significant risk of secondary graft failure and death [18]. Thus, the outcome of this procedure has not been well established in patients with GOF-STAT1 mutations yet.

\section{Conclusion}

The clinical presentations of our patient with severe, early-onset CID support the notion that STAT1 GOF mutations give rise to a wide range of disease phenotypes including fungal and mycobacterial infections, autoimmunity, and combined immunodeficiency. Regardless, the most commonly seen presenting symptom and clinical presentation is that of progressive bronchiectasis. The proper treatment may not stop progressive and devastating lung disease, which in turn may be a detterent to deciding to use HSCT as a treatment option.

\section{Acknowledgements}

The authors are grateful to the patient and her family for their cooperation, and to the scientists from the Laboratory of Human Genetics of Infectious Diseases at the Necker Hospital in Paris for performing molecular tests.

\section{Conflict of interest}

The authors declare no conflict of interest.

\section{References:}

1. Smith MP. Diagnosis and management of bronchiectasis. CMAJ 2017; 189: E828-E835. doi: 10.1503/cmaj.160830

2. Bush A, Floto RA. Pathophysiology, causes and genetics of paediatric and adult bronchiectasis. Respirology. 2019; 24: 1053-1062. doi: 10.1111/resp.13509

3. Yazdani R, Abolhassani $\mathrm{H}$, Asgardoon $\mathrm{MH}$, et al. Infectious and Noninfectious Pulmonary Complications in Patients with Primary Immunodeficiency Disorders. J Investig Allergol Clin Immunol. 2017; 27(4): 213-224. doi: 10.18176/jiaci.0166.

4. Shillitoe B, Bangs C, Guzman D, et al. The United Kingdom Primary Immune Deficiency (UKPID) registry 2012 to 2017. Clin Exp Immunol. 2018; 192(3): 284-291. doi: 10.1111/cei.13125.

5. Liu L, Okada S, Kong XF, et al. Gain-of-function human STAT1 mutations impair IL-17 immunity and underlie chronic mucocutaneous candidiasis. J Exp Med. 2011; 208(8): 1635-1648. doi: 10.1084/jem.20110958

6. Frans G., Moens L., Schaballie H., et al. Gain-of-function mutations in signal transducer and activator of transcription 1 (STAT1): chronic mucocutaneous candidiasis accompanied by enamel defects and delayed dental shedding. J Allergy Clin Immunol. 2014; 134(5): 1209-1213.e6. doi: 10.1016/j. jaci.2014.05.044

7. Dotta L, Scomodon O, Padoan R, et al. Clinical and immunological data of nine patients with chronic mucocutaneous candidiasis disease. Data Brief. 2016; 7: 311-315. doi: 10.1016/j. dib.2016.02.040

8. Breuer O, Daum H, Cohen-Cymberknoh M, et al. Autosomal dominant gain of function STAT1 mutation and severe bronchiectasis. Respir Med. 2017; 126: 39-45. doi: 10.1016/j. rmed.2017.03.018.

9. Huh HJ, Jhun BW, Choi SR, et al. Bronchiectasis and Recurrent Respiratory Infections with a De Novo STAT1 Gain-of-Function Variant: First Case in Korea. Yonsei Med J. 2018; 59(8): 10041007. doi: 10.3349/ymj.2018.59.8.1004

10. Sharfe N, Nahum A, Newell A, et al. Fatal combined immunodeficiency associated with heterozygous mutation in STAT1. J 
Allergy Clin Immunol. 2014; 133(3): 807-817. doi: 10.1016/j. jaci.2013.09.032

11. Baris S, Alroqi F, Kiykim A, et al. Severe Early-Onset Combined Immunodeficiency due to Heterozygous Gain-of-Function Mutations in STAT1. J Clin Immunol. 2016; 36(7): 641-648. doi: 10.1007/s10875-016-0312-3.

12. Eren Akarcan S, Ulusoy Severcan E, Edeer Karaca N, et al. Gain-of-Function Mutations in STAT1: A Recently Defined Cause for Chronic Mucocutaneous Candidiasis Disease Mimicking Combined Immunodeficiencies. Case Reports Immunol. 2017; 2017: 2846928. doi: 10.1155/2017/2846928.

13. Toubiana J, Okada S, Hiller J, et al. International STAT1 Gain-of-Function Study Group. Heterozygous STAT1 gain-of -function mutations underlie an unexpectedly broad clinical phenotype. Blood. 2016; 127(25): 3154-3164. doi: 10.1182/ blood-2015-11-679902.

14. Kobbe R, Kolster M, Fuchs S, et al. Common variable immunodeficiency, impaired neurological development and reduced numbers of T regulatory cells in a 10-year-old boy with a STAT1 gain-of-function mutation. Gene. 2016; 586(2): 234238. doi: 10.1016/j.gene.2016.04.006
15. Uzel G, Sampaio EP, Lawrence MG, et al. Dominant gain-of -function STAT1 mutations in FOXP3 wild-type immune dysregulation-polyendocrinopathy-enteropathy-X-linked-like syndrome. J Allergy Clin Immunol. 2013; 131(6): 1611-1623. doi: 10.1016/j.jaci.2012.11.054.

16. Soltész B, Tóth B, Shabashova N, et al. New and recurrent gain-of -function STAT1 mutations in patients with chronic mucocutaneous candidiasis from Eastern and Central Europe. J Med Genet. 2013; 50(9): 567-578. doi: 10.1136/jmedgenet-2013-101570

17. Sampaio EP, Hsu AP, Pechacek J, et al. Signal transducer and activator of transcription 1 (STAT1) gain-of-function mutations and disseminated coccidioidomycosis and histoplasmosis. J Allergy Clin Immunol. 2013; 131(6): 1624-1634. doi: 10.1016/j.jaci.2013.01.052.

18. Leiding JW, Okada S, Hagin D, et al. Inborn Errors Working Party of the European Society for Blood and Marrow Transplantation and the Primary Immune Deficiency Treatment Consortium. Hematopoietic stem cell transplantation in patients with gain-of-function signal transducer and activator of transcription 1 mutations. J Allergy Clin Immunol. 2018; 141(2): 704-717.e5. doi: 10.1016/j.jaci.2017.03.049. 